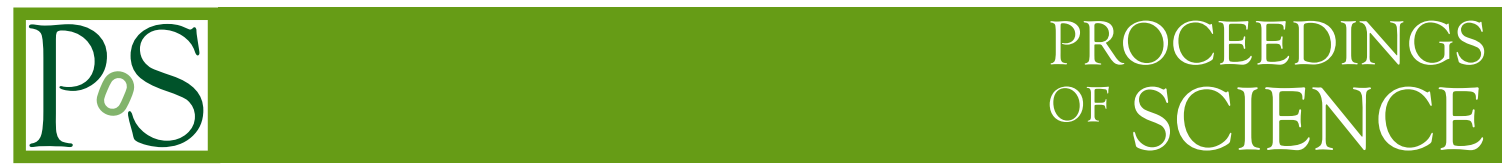

\title{
Rare top quark production in CMS
}

\author{
Nicolas Chanon ${ }^{a, 1, *}$ \\ ${ }^{a}$ Université de Lyon, Université Claude Bernard Lyon 1, CNRS-IN2P3, Institut de Physique Nucléaire de \\ Lyon, Villeurbanne, France \\ E-mail: nicolas.pierre.chanon@cern.ch
}

Recent studies of rare processes involving top quark production at the CMS experiment are presented. We will describe measurements of single top quark production in association with a gauge boson, and the search for four top quark production. The latter process, to which the LHC experiments are starting to be sensitive, can be used to constrain various new physics models, including modified top quark Yukawa coupling.

The Eighth Annual Conference on Large Hadron Collider Physics-LHCP2020

25-30 May, 2020

online

\footnotetext{
${ }^{1}$ For the CMS collaboration.

* Speaker
} 


\section{Introduction}

Very rare processes involving the production of top quarks are opening a wide range of opportunities for testing standard model (SM) predictions. These proceedings will focus on the measurement of single top production in association with a gauge boson $(Z$ or $\gamma)$, and the production of four top quarks at the CMS experiment [1]. These processes are sensitive to modified top - boson couplings $(t Z, t \gamma$ and $t H)$, and to various models of new physics. A comparison of cross sections for these processes with other top quark processes is shown in Fig. 1.

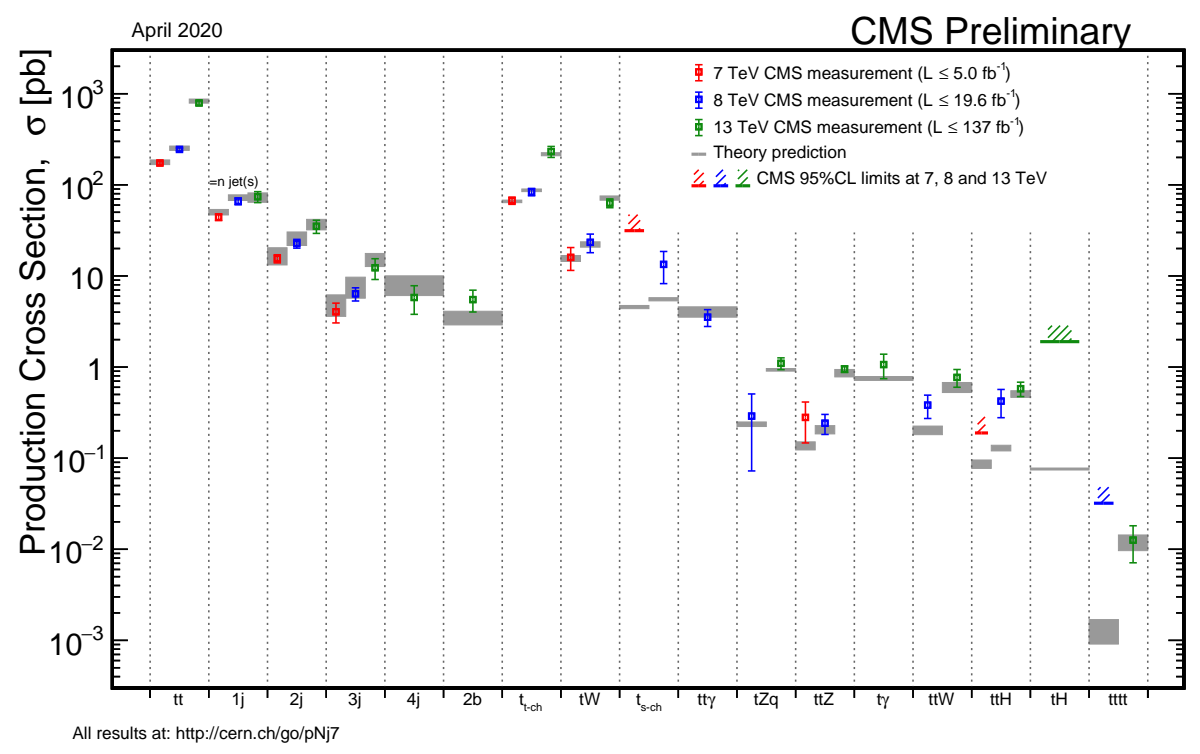

Figure 1: Overview of top quarks measurements at CMS, including $t Z q, t \gamma q$ and $t \bar{t} t \bar{t}$ measurements [2].

\section{Single top quark with a vector boson}

\subsection{Single top quark with a $Z$ boson}

The measurement of the top $+Z$ process [3] is performed in the $3 \ell$ final state with 2016 and 2017 data. Compared to the previous CMS analysis, the rejection of non-prompt lepton background was improved by a factor 4 thanks to an optimized and dedicated lepton identification method. The remaining non-prompt lepton background is measured from data, by measuring the probability for a non-prompt lepton to be identified as a prompt lepton (misidentification ratio) in a QCD sample; the fake ratio is then applied to events with a loosened analysis selection. The signal is extracted from a simultaneous likelihood fit of a BDT discriminator in three categories: one event class targeting the signal and two event classes constraining the $t \bar{t} Z$ background. The BDT uses the presence of a forward jet $(|\eta|<4.7)$ in the event, a peculiar signature belonging to the electroweak production of a single top quark. The main systematic uncertainties are arising from the non-prompt background estimate, jet energy scale, lepton efficiency, final-state radiation and $t Z q$ QCD scale uncertainty. The BDT discriminant used in the fit is shown in Fig. 2. The $t Z q$ process is observed at $8.2 \sigma(7.7 \sigma$ expected). 

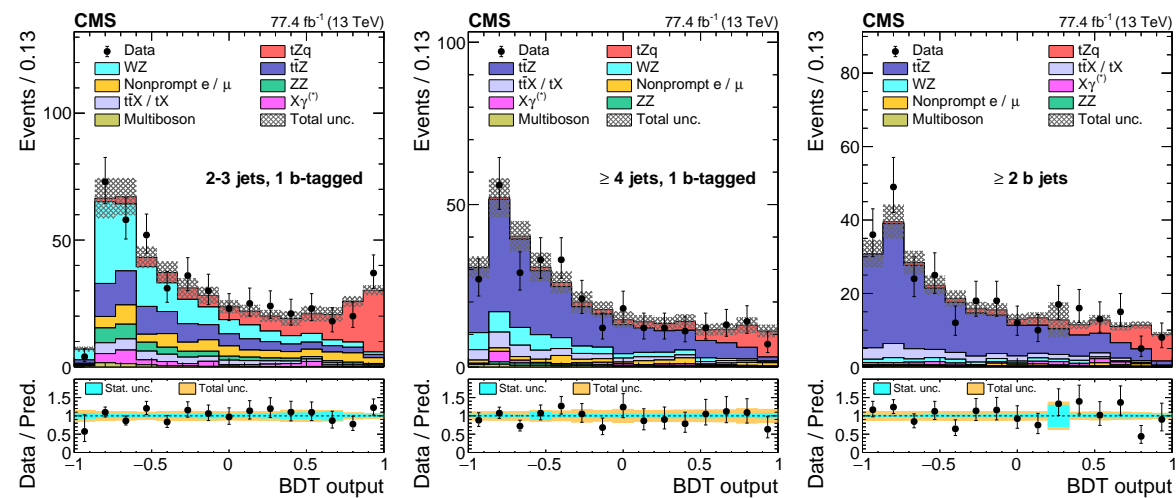

Figure 2: BDT discriminator distributions used for signal extraction in $t Z q$ analysis [3], left: in the signal region; centre and right: in the control region targeting $t \bar{t} Z$ background.

\subsection{Single top quark with a photon}

In the $t \gamma q$ analysis [4], the dominant background is $t \bar{t}+\gamma$, which is constrained in a control region with an additional identified b-tagged jet. The jet faking photon background is also important and must be measured. A misidentification ratio method is used, consisting in relaxing photon identification requirements to measure the probability of mis-identifying a non-prompt photon as a prompt photon. Similarly to the $t Z q$ analysis, the single top + photon analysis makes use of the forward jet, whose pseudorapidity is included as an input variable to a BDT. The BDT output is shown in Fig. 4. A simultaneous fit of $t \gamma q$ and $t \bar{t} \gamma$ regions is performed, which led to the first evidence for the $t \gamma q$ process with a significance of $4.4 \sigma$ (3.0 $\sigma$ expected).

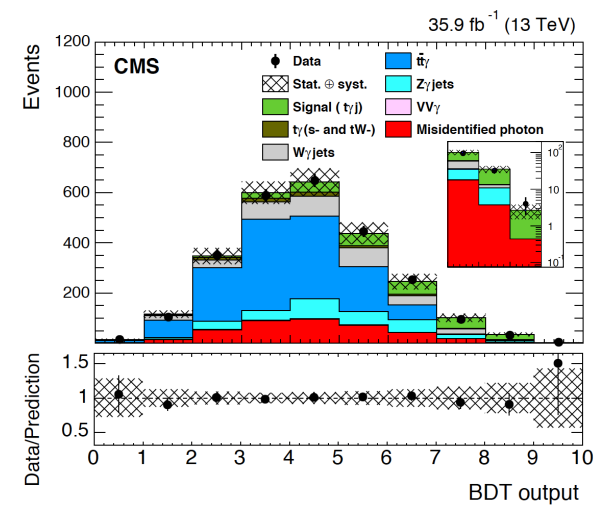

Figure 3: BDT output distribution in $t \gamma q$ measurement [4] after performing the fit.

\section{Four top quark production}

The four top quark production process is predicted in the SM with the smallest cross section among the processes previously presented in these proceedings, $12.0 \mathrm{fb}$ (predicted in NLO QCD with electroweak corrections). Four top quark production was recently searched for in single lepton and dilepton channels [6], but this analysis suffers from the overwhelming hadronic backgrounds 
among which the poorly known $t \bar{t}+b \bar{b}$ process. These proceedings will focus on the multilepton channel.

The measurement is performed using the full Run 2 dataset in $2 \ell s s$ and $\geq 3 \ell$ final states [5]. Taking advantage of the large multiplicity of jets in the event, $H T>300 \mathrm{GeV}$ is required (where $H T$ is the scalar $p_{T}$ sum of all reconstructed objects in the event). Events are classified with a BDT in 17 signal regions, with a sequential cross-check analysis classifying events according to the number of jets and identified b-tagged jets. In addition, control regions targeting $t t V$ background are used in the likelihood fit. Charge misidentification ( $2 \ell s s$ category only) and non-prompt lepton background are measured from data. The number of events with additional jets in $t \bar{t}+W / Z$ simulation events is corrected for the increase in ISR/FSR radiation observed in $t \bar{t}$ measurements, while $t \bar{t}+H / W / Z$ simulation events are corrected for the $70 \%$ increase in $t \bar{t}+b \bar{b}$ production observed in data relative to the SM. The main systematic uncertainty sources are jet energy scale, b-tagging, the modeling of $t \bar{t}+b \bar{b}$ and initial/final state radiation. The observed significance is $2.6 \sigma$ (2.7 $\sigma$ expected). The $t \bar{t} t \bar{t}$ production is involving virtual Higgs boson diagrams, thus its cross section can be used to probe the top-Higgs Yukawa coupling $y_{t}$. A value of $\left|y_{t} / y_{t}^{S M}\right|<1.7$ at $95 \%$ C.L. is found (Fig. 4); it is complementary to, but not as precise as, measurements of $\kappa_{t}$ in direct Higgs boson measurements.

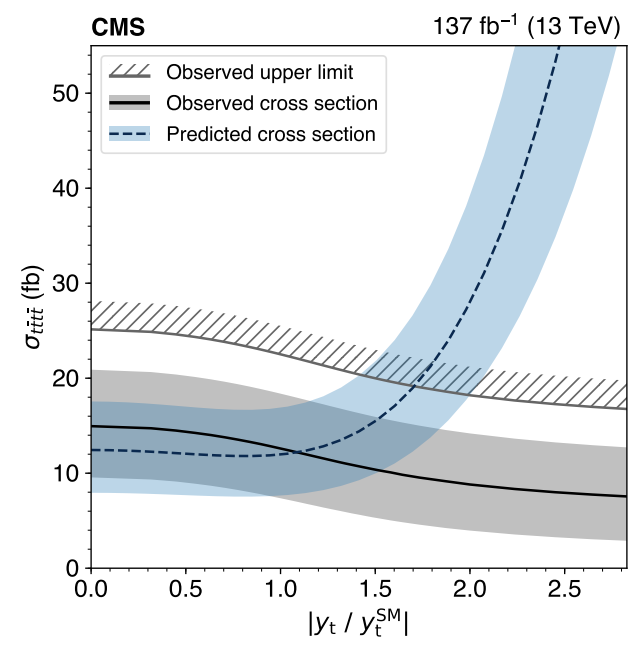

Figure 4: Limits on the cross section for four top production as a function of the top Yukawa coupling [5].

\section{Conclusions}

The $t Z q$ process was observed at CMS thanks to an improved lepton identification that permitted to reduce the non-prompt lepton background. This channel is now mature for differential cross section measurements. The first evidence for the $t \gamma q$ process has been obtained, the next goal being the observation. The four top quark process is very rare and is not yet observed, although results are approaching the evidence. Searches for this production process and interpretations beyond the SM will be pursued at the LHC Run 3. 


\section{References}

[1] CMS Collaboration, JINST 3 (2008) S08004.

[2] CMS Collaboration, https://twiki.cern.ch/twiki/bin/view/CMSPublic/PhysicsResultsCombined

[3] CMS Collaboration, Phys. Rev. Lett. 122 (2019) no.13, 132003

[4] CMS Collaboration, Phys. Rev. Lett. 121 (2018) no.22, 221802

[5] CMS Collaboration, Eur. Phys. J. C 80 (2020) no.2, 75

[6] CMS Collaboration, JHEP 11 (2019), 082 